\title{
Prevalence and antimicrobial sensitivity of bacterial isolates from canine wound infection in Tandojam, Sindh
}

Dildar Hussain Kalhoro ${ }^{1 *}$, Mansoor Ibrahim Ansari ${ }^{1}$, Shahid Hussain Abro $^{1}$, Muhammad Saleem Kalhoro ${ }^{1}$, Ahmed Nawaz Tuino ${ }^{1}$, Shahnawaz Kumbhar ${ }^{1}$, Asmatullah Kaka ${ }^{1}$, Ghulam Murtaza Lochi ${ }^{1}$, Muhammad Qasim Mazari ${ }^{1}$ and Muhammad Ali Chandio ${ }^{2}$

1. Faculty of Animal Husbandry and Veterinary Sciences, Sindh Agriculture University, Tandojam-70060-Pakistan

2. Shaheed Benazir Bhutto University of Veterinary and Animal Sciences, Sakrand-Pakistan

*Corresponding author's email: drdildarkalhoro@gmail.com

Citation

Dildar Hussain Kalhoro, Mansoor Ibrahim Ansari, Shahid Hussain Abro, Muhammad Saleem Kalhoro, Ahmed Nawaz Tuino, Shahnawaz Kumbhar, Asmatullah Kaka, Ghulam Murtaza Lochi, Muhammad Qasim Mazari and Muhammad Ali Chandio. Prevalence and antimicrobial sensitivity of bacterial isolates from canine wound infection in Tandojam, Sindh. Pure and Applied Biology. Vol. 8, Issue 1, pp372-379.

http://dx.doi.org/10.19045/bspab.2018.700196

\begin{tabular}{llll}
\hline \hline Received: 19/09/2018 & Revised: 20/11/2018 & Accepted: 27/11/2018 & Online First: 03/12/2018 \\
\hline \hline
\end{tabular}

\section{Abstract}

A total of 100 canine wound samples of accidental wound $(n=40)$, pyoderma $(n=16)$, tail wound $(n=15)$, localized anal wound $(n=10)$, otitis $(n=8)$, lacerated mammary wound $(n=6)$ and postsurgical wound $(n=5)$ were taken from Clinic of Surgery and Obstetrics and brought to the Department of Veterinary Microbiology, Faculty of Animal Husbandry and Veterinary Sciences, Sindh Agriculture University, Tandojam to isolate and identify bacterial species responsible for canine wound infection and their susceptibility to various antibiotics. Samples were confirmed through morphological, culture and biochemical tests. Staphylococcus aureus (67\%) was found to be the predominant isolate. It was followed by Pseudomonas aeruginosa (50\%), Escherichia coli (40\%), Streptococcus pyogenes and Pasteurella canis (38\%), Bacillus cereus (36\%) and Micrococcus luteus (25\%). Staphylococcus aureus was isolated and identified as 40 (55\%), 10 (90\%), 8(62.5\%), 6(66.6\%), 15(86.6\%), 16(62.5\%) and 5(80\%) from accidental wound, localized anal wound, otitis, lacerated mammary wound, tail wound, pyoderma and post- surgical wounds respectively. Antibiotics Erythromycin and Ofloxacin were sensitive to S. aureus. B. cereus was sensitive to Ampicillin and Gentamicin. P. canis was sensitive to Ampicillin, Enrofloxacin and Ofloxacin. E. coli was sensitive to Enrofloxacin and Ofloxacin. M. luteus was sensitive to the Ampicillin and Enrofloxacin. P. aeruginosa and S. pyogenes were sensitive to Enrofloxacin.

Keywords: Antibiotic; Canine; Sensitivity; Staphylococcus; Wound

\section{Introduction}

Bacterial wound infection is a critical problem and the treatment of wound infections remain a significant concern in dogs. Wounds can be classified as acute and chronic. The acute type of wounds either upsetting, distressing or surgically treated through the healing process but chronic 
wounds are not resolved over a long time [1]. It is reported that bacterial infections persist more than $80 \%$ are likely to involve biofilms [2]. Biofilm bacteria are resistant to antimicrobial agents and host immune responses that results in chronic infection [3]. The bacteria adhere to the surface of wound by attaching to the exposed extracellular matrix, multiply and develop into microcolonies, these biofilms once established, resist the action of antimicrobials and evade the host defense mechanism [4]. Most dermal wounds are populated with aerobic and anaerobic microorganisms that occur mainly from mucosal surfaces such as oral cavity and gut. Wound due to bacterial infection and resistance to antibiotics are the common post-surgical and scientific challenges. Wounds of bacterial contaminations are the frequent hospital acquired infections causing more than $80 \%$ mortality [5]. Escherichia, Enterococci, Klebsiella, Pseudomonas, Enterobacter, Proteus and Acinetobacter are the most frequent bacterial genera infecting wounds $[6,7]$. Wound infections are identified as the most unfavorable problem especially in the presence of foreign materials that increases the risk of serious infection even with relatively minute bacterial infection [8].

Antimicrobial resistance is a very complex problem involving various bacterial species, resistance mechanisms, transfer mechanisms and reservoirs. Research have revealed that antimicrobial use in pet animals contributes to the selection of antimicrobial resistant and possess threat to the people because of transmission of resistant zoonotic microorganism via the food chain and oblique switch of resistant genes from animals to man [9]. Resistant bacteria isolated from pets are observable by using their absence as key organisms which have been reported to understand resistance gene organization [10]. For example, methicillin resistance Staphylococcus aureus of canine origin may be more common than reported [11]. Excessive use of antibiotics together with the length of time cause a significant antibiotic resistance to wound infecting bacteria [12] that later increase the problems and costs of treatment [13].

Applications for proper preventive measures to control antimicrobial resistant microorganisms and surveillance protocols which include patterns of antimicrobial resistant in human are the key component in decreasing rate of infection. Bacterial culture and antimicrobial susceptibility testing of clinical site of surgical infections (SSIs) in veterinary practice is essential for the diagnosis of bacterial infection and to guide the therapy towards rational antimicrobial use. Culture based treatments also help to facilitate surveillance efforts [14]. Surgical procedures are successful with early decontamination and tension free closure in companion animals [15]. Therefore, present study is planned to evaluate the microbial profile of wounds in dogs and select appropriate drug for their better management practices.

\section{Materials and methods Isolation and Identification of microbes}

A total of 100 canine wound samples of accidental wound $(n=40)$, pyoderma $(n=16)$, tail wound $(\mathrm{n}=15)$, localized anal wound $(n=10)$, otitis $(n=8)$, lacerated mammary wound $(n=6)$ and post-surgical wound $(n=5)$ were taken from Clinic of Surgery and Obstetrics and brought to the Department of Veterinary Microbiology, Faculty of Animal Husbandry and Veterinary Sciences, Sindh Agriculture University, Tandojam. Samples were processed for the bacterial culture by inoculating swabs into agar plates. i.e Nutrient agar, Maconkey's agar, Blood agar and incubated at $37^{\circ} \mathrm{C}$ for $24 \mathrm{~h}$ to separate the colonies for each kind of bacteria present in culture. Morphological characteristics were noted and confirmation of morphological difference among them was done by Gram 
staining. Biochemical Tests such as Catalase, Oxidase, Coagulase, Indole, Triple Sugar Iron Test, Simmon Citrate Test, Voges Proskauer Test, Nitrate Reduction Test were done for further identification.

\section{Antibiotic sensitivity test}

The antibiotic sensitivity test was performed by method described by [16]. Pure colonies were taken by wire loop and inoculated in to $5 \mathrm{ml}$ normal saline. Sterile cotton swab was taken and dipped into the suspension and rotated several times with a firm pressure on the inside wall of the tube. Sterile swab was streaked over the entire surface of Muller Hinton agar in three different directions to make sure an even distribution of the inoculum. After 3-5 minutes of drying, the antibiotic disks were placed there. Plates were incubated for 24 hours at $37^{\circ} \mathrm{C}$. The inhibitory zone diameters gained around the discs were measured after incubation for $24 \mathrm{~h}$ at $37^{\circ} \mathrm{C}$ and evaluated. Following antibiotics were used: Ampicillin, Amikacin, Erythromycin, Enrofloxacin, Gentamicin, Kanamycin, Neomycin, Ofloxacin, Penicillin, Streptomycin and Tetracycline.

\section{Results}

\section{Prevalence of bacterial isolates from canine wound}

Results of the bacterial isolates from canine wound are described in (Table 1). The predominant isolate from different kinds of wound infection of dogs was found to be Staphylococcus aureus (67\%). It was followed by Pseudomonas aeruginosa (50\%), Escherichia coli (40\%), Streptococcus pyogenes and Pasteurella canis (38\%), Bacillus cereus (36\%) and Micrococcus luteus (25\%). S. aureus was isolated and identified as $40(55 \%), 10$ (90\%), 8(62.5\%), 6(66.6\%), 15(86.6\%), $16(62.5 \%)$ and $5(80 \%)$ from accidental wound, localized anal wound, otitis, lacerated mammary wound, tail wound, pyoderma and post-surgical wound respectively. $P$. aeruginosa became a main cause of infection in majority of wounds except otitis in which the E. coli and P. canis had been predominant. In addition, $M$. luteus was observed to be least in all the wounds except otitis where $S$. pyogenes become least predominant. Prevalence of isolated bacterial species from canine wound is summarized in (Table 1).

\section{Antibiotics sensitivity}

The antibiotics sensitivity against bacterial isolates from canine wound is presented in (Table 2).

\section{Staphylococcus aureus}

Erythromycin and Ofloxacin were highly sensitive against Staphylococcus aureus. Ampicillin, Amikacin, Enrofloxacin, Penicillin, Gentamicin, Streptomycin and tetracycline were quite sensitive. Kanamycin was moderately sensitive. Neomycin was not sensitive against this bacterial specie.

\section{Bacillus cereus}

Ampicillin and Gentamicin were highly sensitive against Bacillus cereus. Amikacin, Erythromycin, Penicillin and Streptomycin were quite sensitive. Kanamycin, Neomycin, Ofloxacin and Tetracycline were moderately sensitive. Enrofloxacin was not sensitive against Bacillus cereus.

\section{Pasteurella canis}

Ampicillin, Enrofloxacin and Ofloxacin were highly sensitive against Pasteurella canis. Amikacin, Erythromycin, Gentamicin, Kanamycin, Neomycin and streptomycin were found quite sensitive. Erythromycin, Penicillin and Tetracycline were not sensitive against Pasteurella canis.

\section{Escherichia coli}

Enrofloxacin and Ofloxacin were highly sensitive against Escherichia coli. Ampicillin, Amikacin, Erythromycin, Gentamicin, Streptomycin and Tetracycline were quite sensitive. Kanamycin and Neomycin were moderately sensitive. Penicillin was not sensitive against Escherichia coli. 

Kalhoro et al.

Table 1. Prevalence of bacterial isolates from canine wound

\begin{tabular}{|c|c|c|c|c|c|c|c|c|}
\hline Type of wound & $\begin{array}{c}\text { No of } \\
\text { sample }\end{array}$ & $\begin{array}{c}\text { S. } \\
\text { aureus }\end{array}$ & B. cereus & $\boldsymbol{E . ~ c o l i}$ & M. luteus & $\begin{array}{c}\boldsymbol{P .} \\
\text { aeruginosa }\end{array}$ & $\begin{array}{c}\text { S. } \\
\text { pyogenes }\end{array}$ & P. canis \\
\hline Accidental wound & 40 & $22(55 \%)$ & $09(22.5 \%)$ & $12(30 \%)$ & $07(17.5 \%)$ & $14(35 \%)$ & $13(32.5 \%)$ & $08(20 \%)$ \\
\hline Pyoderma & 16 & $\begin{array}{c}10 \\
(62.5 \%)\end{array}$ & $08(50 \%)$ & $06(37.5 \%)$ & $05(31.2 \%)$ & $10(62.5 \%)$ & $06(37.5 \%)$ & $07(43.7 \%)$ \\
\hline Tail wound & 15 & $\begin{array}{c}13 \\
(86.6 \%)\end{array}$ & $08(53.3 \%)$ & $07(46.6 \%)$ & $04(26.6 \%)$ & $11(73.3 \%)$ & $05(33.3 \%)$ & $07(46.6 \%)$ \\
\hline $\begin{array}{c}\text { Localized anal } \\
\text { wound }\end{array}$ & 10 & $09(90 \%)$ & $04(40 \%)$ & $06(60 \%)$ & $03(30 \%)$ & $06(60 \%)$ & $07(70 \%)$ & $04(40 \%)$ \\
\hline Otitis & 08 & $05(62.5)$ & $03(37.5 \%)$ & $05(62.5 \%)$ & $04(50 \%)$ & $04(50 \%)$ & $02(25 \%)$ & $05(62.5 \%)$ \\
\hline $\begin{array}{c}\text { Lacerated } \\
\text { mammary wound }\end{array}$ & 06 & $\begin{array}{c}04 \\
(66.6 \%)\end{array}$ & $02(33.3 \%)$ & $02(33.3 \%)$ & $01(16 \%)$ & $02(33.3 \%)$ & $03(50 \%)$ & $05(83.3 \%)$ \\
\hline $\begin{array}{c}\text { Postsurgical } \\
\text { wound }\end{array}$ & 05 & $04(80 \%)$ & $02(40 \%)$ & $02(40 \%)$ & $01(20 \%)$ & $03(60 \%)$ & $02(40 \%)$ & $02(40 \%)$ \\
\hline Total & 100 & $67(67 \%)$ & $36(36 \%)$ & $40(40 \%)$ & $25(25 \%)$ & $50(50 \%)$ & $38(38 \%)$ & $38(38 \%)$ \\
\hline
\end{tabular}

\section{Micrococcus luteus}

Ampicillin and Enrofloxacin were highly sensitive against Micrococcus luteus. Amikacin, Gentamicin, Kanamycin, Neomycin, Penicillin, Streptomycin and Tetracycline were quite sensitive against this bacterial specie. Ofloxacin and Erythromycin were not sensitive against Micrococcus luteus.

\section{Pseudomonas aeruginosa}

Enrofloxacin was highly sensitive against Pseudomonas aeruginosa. Ampicillin, Amikacin, Erythromycin, Gentamicin,
Kanamycin, Neomycin, Ofloxacin, Streptomycin were quite sensitive. Tetracycline was found moderately sensitive. Penicillin was not sensitive against Pseudomonas aeruginosa.

\section{Streptococcus pyogenes}

Enrofloxacin was highly sensitive against Streptococcus pyogenes. Amikacin, Gentamicin, Neomycin, Ofloxacin Penicillin, Streptomycin and Tetracycline, were quite sensitive. Ampicillin and Erythromycin were moderately sensitive. Kanamycin was not sensitive against Streptococcus pyogenes.

Table 2. The antibiotic sensitivity of bacterial isolates from canine wound

\begin{tabular}{|c|c|c|c|c|}
\hline Bacterial species & $\begin{array}{c}\text { Antibiotic discs } \\
\text { used }\end{array}$ & $\begin{array}{c}\text { Zone around } \\
\text { Discs }\end{array}$ & $\begin{array}{c}\text { Identification } \\
\text { of sensitivity }\end{array}$ & Degree of sensitivity \\
\hline \multirow{5}{*}{ Staphylococcus aureus } & Ampicillin & $10 \mathrm{~mm}$ & +++ & Quite sensitive \\
& Amikacin & $08 \mathrm{~mm}$ & +++ & Quite sensitive \\
& Erythromycin & $14 \mathrm{~mm}$ & ++++ & Highly sensitive \\
& Enrofloxacin & $08 \mathrm{~mm}$ & +++ & Quite sensitive \\
& Gentamicin & $10 \mathrm{~mm}$ & +++ & Quite sensitive \\
& Kanamycin & $04 \mathrm{~mm}$ & ++ & Moderately sensitive \\
& Neomycin & $0 \mathrm{~mm}$ & - & No sensitivity \\
& Ofloxacin & $15 \mathrm{~mm}$ & ++++ & Highly sensitive \\
& Penicillin & $10 \mathrm{~mm}$ & +++ & Quite sensitive \\
& Streptomycin & $08 \mathrm{~mm}$ & ++ & Quite sensitive \\
& Tetracycline & $10 \mathrm{~mm}$ & +++ & Quite sensitive \\
\hline \multirow{5}{*}{ Bacillus cereus } & Ampicillin & $15 \mathrm{~mm}$ & ++++ & Highly sensitive \\
& Amikacin & $10 \mathrm{~mm}$ & +++ & Quite sensitive \\
& Erythromycin & $08 \mathrm{~mm}$ & +++ & Quite sensitive \\
& Enrofloxacin & $0 \mathrm{~mm}$ & - & No sensitivity \\
& Gentamicin & $15 \mathrm{~mm}$ & ++++ & Highly sensitive \\
& Kanamycin & $05 \mathrm{~mm}$ & ++ & Moderately sensitive \\
\hline
\end{tabular}




\begin{tabular}{|c|c|c|c|c|}
\hline & $\begin{array}{c}\text { Neomycin } \\
\text { Ofloxacin } \\
\text { Penicillin } \\
\text { Streptomycin } \\
\text { Tetracycline }\end{array}$ & $\begin{array}{l}04 \mathrm{~mm} \\
04 \mathrm{~mm} \\
08 \mathrm{~mm} \\
08 \mathrm{~mm} \\
05 \mathrm{~mm}\end{array}$ & $\begin{array}{c}+++ \\
++ \\
++ \\
++ \\
++\end{array}$ & $\begin{array}{c}\text { Moderately sensitive } \\
\text { Moderately sensitive } \\
\text { Quite sensitive } \\
\text { Quite sensitive } \\
\text { Moderately sensitive }\end{array}$ \\
\hline Pasteurella canis & $\begin{array}{l}\text { Ampicillin } \\
\text { Amikacin } \\
\text { Erythromycin } \\
\text { Enrofloxacin } \\
\text { Gentamicin } \\
\text { Kanamycin } \\
\text { Neomycin } \\
\text { Ofloxacin } \\
\text { Penicillin } \\
\text { Streptomycin } \\
\text { Tetracycline }\end{array}$ & $\begin{array}{c}16 \mathrm{~mm} \\
09 \mathrm{~mm} \\
0 \mathrm{~mm} \\
13 \mathrm{~mm} \\
09 \mathrm{~mm} \\
09 \mathrm{~mm} \\
08 \mathrm{~mm} \\
14 \mathrm{~mm} \\
00 \mathrm{~mm} \\
10 \mathrm{~mm} \\
0 \mathrm{~mm}\end{array}$ & $\begin{array}{c}++++ \\
+++ \\
- \\
++++ \\
+++ \\
+++ \\
+++ \\
++++ \\
- \\
+++ \\
-\end{array}$ & $\begin{array}{c}\text { Highly sensitive } \\
\text { Quite sensitive } \\
\text { No sensitivity } \\
\text { Highly sensitive } \\
\text { Quite sensitive } \\
\text { Quite sensitive } \\
\text { Quite sensitive } \\
\text { Highly sensitive } \\
\text { No sensitivity } \\
\text { Quite sensitive } \\
\text { No sensitivity }\end{array}$ \\
\hline Escherichia coli & $\begin{array}{l}\text { Ampicillin } \\
\text { Amikacin } \\
\text { Erythromycin } \\
\text { Enrofloxacin } \\
\text { Gentamicin } \\
\text { Kanamycin } \\
\text { Neomycin } \\
\text { Ofloxacin } \\
\text { Penicillin } \\
\text { Streptomycin } \\
\text { Tetracycline } \\
\end{array}$ & $\begin{array}{l}08 \mathrm{~mm} \\
08 \mathrm{~mm} \\
08 \mathrm{~mm} \\
14 \mathrm{~mm} \\
10 \mathrm{~mm} \\
04 \mathrm{~mm} \\
04 \mathrm{~mm} \\
15 \mathrm{~mm} \\
0 \mathrm{~mm} \\
08 \mathrm{~mm} \\
08 \mathrm{~mm} \\
\end{array}$ & $\begin{array}{c}++ \\
+++ \\
+++ \\
++++ \\
+++ \\
++ \\
++ \\
++++ \\
- \\
+++ \\
+++ \\
\end{array}$ & $\begin{array}{c}\text { Quite sensitive } \\
\text { Quite sensitive } \\
\text { Quite sensitive } \\
\text { Highly sensitive } \\
\text { Quite sensitive } \\
\text { Moderate sensitive } \\
\text { Moderate sensitive } \\
\text { Highly sensitive } \\
\text { No sensitivity } \\
\text { Quite sensitive } \\
\text { Quite sensitive }\end{array}$ \\
\hline Micrococcus luteus & $\begin{array}{l}\text { Ampicillin } \\
\text { Amikacin } \\
\text { Erythromycin } \\
\text { Enrofloxacin } \\
\text { Gentamicin } \\
\text { Kanamycin } \\
\text { Neomycin } \\
\text { Ofloxacin } \\
\text { Penicillin } \\
\text { Streptomycin } \\
\text { Tetracycline }\end{array}$ & $\begin{array}{l}15 \mathrm{~mm} \\
10 \mathrm{~mm} \\
00 \mathrm{~mm} \\
15 \mathrm{~mm} \\
08 \mathrm{~mm} \\
08 \mathrm{~mm} \\
08 \mathrm{~mm} \\
0 \mathrm{~mm} \\
10 \mathrm{~mm} \\
08 \mathrm{~mm} \\
10 \mathrm{~mm}\end{array}$ & $\begin{array}{c}++++ \\
+++ \\
- \\
++++ \\
+++ \\
+++ \\
+++ \\
- \\
+++ \\
+++ \\
+++\end{array}$ & $\begin{array}{l}\text { Highly sensitive } \\
\text { Quite sensitive } \\
\text { No sensitivity } \\
\text { Highly sensitive } \\
\text { Quite sensitive } \\
\text { Quite sensitive } \\
\text { Quite sensitive } \\
\text { No sensitivity } \\
\text { Quite sensitive } \\
\text { Quite sensitive } \\
\text { Quite sensitive }\end{array}$ \\
\hline $\begin{array}{c}\text { Pseudomonas } \\
\text { aeruginosa }\end{array}$ & $\begin{array}{l}\text { Ampicillin } \\
\text { Amikacin } \\
\text { Erythromycin } \\
\text { Enrofloxacin } \\
\text { Gentamicin } \\
\text { Kanamycin } \\
\text { Neomycin } \\
\text { Ofloxacin } \\
\text { Penicillin } \\
\text { Streptomycin } \\
\text { Tetracycline }\end{array}$ & $\begin{array}{l}08 \mathrm{~mm} \\
10 \mathrm{~mm} \\
08 \mathrm{~mm} \\
14 \mathrm{~mm} \\
08 \mathrm{~mm} \\
08 \mathrm{~mm} \\
08 \mathrm{~mm} \\
08 \mathrm{~mm} \\
00 \mathrm{~mm} \\
08 \mathrm{~mm} \\
07 \mathrm{~mm}\end{array}$ & $\begin{array}{c}+++ \\
+++ \\
+++ \\
++++ \\
+++ \\
+++ \\
+++ \\
+++ \\
- \\
+++ \\
++\end{array}$ & $\begin{array}{c}\text { Quite sensitive } \\
\text { Quite sensitive } \\
\text { Quite sensitive } \\
\text { Highly sensitive } \\
\text { Quite sensitive } \\
\text { Quite sensitive } \\
\text { Quite sensitive } \\
\text { Quite sensitive } \\
\text { No sensitive } \\
\text { Quite sensitive } \\
\text { Moderately sensitive }\end{array}$ \\
\hline
\end{tabular}




\begin{tabular}{|c|c|c|c|c|}
\hline & Ampicillin & $05 \mathrm{~mm}$ & ++ & Moderately sensitive \\
& Amikacin & $10 \mathrm{~mm}$ & +++ & Quite sensitive \\
& Erythromycin & $04 \mathrm{~mm}$ & ++ & Moderately sensitive \\
& Enrofloxacin & $15 \mathrm{~mm}$ & ++++ & Quite sensitive \\
& Gentamicin & $08 \mathrm{~mm}$ & +++ & No sensitivity \\
Streptococcus & Kanamycin & $0 \mathrm{~mm}$ & - & Quite sensitive \\
& Neomycin & $10 \mathrm{~mm}$ & +++ & Quite sensitive \\
& Ofloxacin & $11 \mathrm{~mm}$ & +++ & Quite sensitive \\
& Penicillin & $08 \mathrm{~mm}$ & +++ & Quite sensitive \\
& Streptomycin & $08 \mathrm{~mm}$ & +++ & +++ \\
& Tetracycline & $08 \mathrm{~mm}$ & & +
\end{tabular}

\section{Discussion}

Rearing of dogs and other pet animals has become increasingly popular in modern society. In this context it is important to note that respiratory diseases pose a major health problem in dogs and other pet animals [17, 18]. Bacteria involved in respiratory infections in dogs and cats are usually Escherichia coli, Klebsiella spp., Streptococcus spp. and Staphylococcus spp [19].

In our study the most dominant isolates from accidental wound, pyoderma, tail wound, localized anal wound, otitis, lacerated mammary wound and post-surgical wounds was $S$. aureus followed by Pseudomonas aeruginosa, Escherichia coli, Streptococcus pyogenes, Pasteurella canis, Bacillus cereus and Micrococcus luteus. Similar results are supported by other authors [20] who reported Staphylococcus intermedius (20\%), Enterococcus spp. (15\%), Coagulasenegative staphylococci (13\%), Escherichia coli $(13 \%)$ as most common aerobic isolates. Whereas, [21] reported Staphylococcus intermedius (23\%), Escherichia coli (18\%), non-lactose fermenting coliforms (14\%) and Pseudomonas spp (14\%) as the most commonly pathogenic isolates from wound infections. While, [22] reported the higher prevalence of Pasteurella canis and Staphylococcus aureus. This is in accordance with our finding in which Staphylococcus aureus and Pasteurella canis were most common. In our study Staphylococcus aureus $(67 \%)$ was found to be the predominant isolate. It was followed by Pseudomonas aeruginosa (50\%), Escherichia coli (40\%), Streptococcus pyogenes and Pasteurella canis (38\%), Bacillus cereus (36\%) and Micrococcus luteus (25\%). Staphylococcus aureus was isolated and identified in 40 (55\%), 10 (90\%), 8(62.5\%), 6(66.6\%), $15(86.6 \%), 16(62.5 \%)$ and $5(80 \%)$ samples from accidental wound, localized anal wound, otitis, lacerated mammary wound, tail wound, pyoderma and post-surgical wound respectively. This is similar to previous reports by [23] who reported higher prevalence of Staphylococcus aureus (33\%), Escherichia coli (18.9\%), Klebsiella pneumonia (16.7\%), Proteus vulgaris (11.4\%), Streptococcus spp (9.2\%) and Pseudomonas aeruginosa (9.6\%) in dogs.

A study by [24] reported that the isolates Staphylococcus intermedius, Staphylococcus aureus, Pseudomonas aeruginosa, Escherichia coli, Pasteurella spp, Corynebacterium spp and bacillus spp. are persistently causative agent of wound infection in dogs. This suggests that canine wounds are polymicrobial in nature and containing pathogens which are significantly prevalent in chronic wounds. In our study Staphylococcus was found in synergistic relation with other bacteria in infected wounds. This agrees with the findings of previous studies [1]. Whereas, [25] reported that bacteria like Staphylococcus never occurs singly and commonly found synergistically with others. The synergism of bacteria might lead to greater pathogenesis 
than individual inputs. This is in accordance with [24] who reported that the Staphylococcus $(65.7 \%)$ was found to be the most predominant isolate from traumatic wounds, while Staphylococcus intermedius as most prevalent bacteria in dog infected wounds [26]. The increased prevalence of Staphylococcus intermedius might be due to occurrence as commensal on skin and mucus membranes. Pseudomonas aeruginosa (75\%) was found to be the main agent isolated from postsurgical wounds which show the similarity with previous reviews [27]. The genus Staphylococcus was most commonly isolated in all type of wounds. This is much like preceding reports in human and equines [28]. The reason for above outcomes may be the microbial isolates have strong affinity for that specific site. The composition and variation of microbial agents in wounds are affected by many factors such as depth of affected tissue, localization, tissue perfusion, intensity, quality and strength of host antimicrobial immune response [25].

Antimicrobial resistance is a critical health problem in companion animals with systemic antimicrobial therapy driving development of AMR across the host spectrum [29, 30]. The antibiotics are mostly resistant due to misuse of prescribed medication [31, 32]. In our study erythromycin and ofloxacin were found highly sensitive against Staphylococcus aureus. Ampicillin and Gentamicin were found highly sensitive against Bacillus cereus. Ampicillin, Enrofloxacin and Ofloxacin were found highly sensitive against Pasteurella canis. Enrofloxacin and Ofloxacin were found highly sensitive against Escherichia coli. Ampicillin and Enrofloxacin were found highly sensitive against Micrococcus luteus. Pseudomonas aeruginosa and Streptococcus pyogenes were observed as highly sensitivity to Enrofloxacin. These results are in agreement with results obtained by [33] who showed that Gentamicin was the most sensitive antibiotic followed by Enrofloxacin, Ciprofloxacin and Amoxicillin. While [34] found that Staphylococcus spp. were predominant and sensitive to Gentamicin and Enrofloxacin but were generally resistant to Lincomycin and Oxytetracycline. whereas, [35] investigated that Escherichia coli, Proteus spp, Staphylococcus aureus and Streptococcus pyogenes were major isolates from canine infections. Majority of the isolates were highly sensitive to Gentamicin. Lowered sensitivity was noticed towards Ampicillin. Gentamicin, Chloramphenicol, Cefixime, Ciprofloxacin were sensitive against Escherichia coli, whereas, Amikacin, Tetracycline, Kanamycin, Vancomycin, Erythromycin and Novobiocin showed their infectivity against these isolates [36]. Thus, our findings are in agreement with above workers.

\section{Conclusion}

It was concluded from present study that the maximum prevalence of canine wound infection is recorded from accidental wound, pyoderma, tail wound, localized anal wound, otitis, lacerated mammary wound and postsurgical wounds. Staphylococcus aureus was predominant specie followed by Escherichia coli, Pseudomonas aeruginosa, Bacillus cereus, Streptococcus pyogenes, Pasteurella canis and Micrococcus luteus, respectively. Antibiotics should be taken into consideration for effective treatment of canine wound infections.

\section{Authors' contributions}

Conceived and designed the experiments: DH Kalhoro, SH Abro \& AN Tuino, Performed the experiments: DH Kalhoro \& MI Ansari, Analyzed the data: MS Kalhoro, GM Lochi \& MA Chandio, Contributed materials/ analysis/ tools: SH Abro, A Kaka \& S Kumbhar, Wrote the paper: DH Kalhoro \& MQ Mazari.

\section{References}

1. Bowler PG, Duerden BI \& Armstrong DG (2001). Wound microbiology and 
associated approaches to wound management. Clin Microbiol Rev 14(2): 244-269.

2. Widgerow AD (2008). Persistence of the chronic wound-implicating biofilm chronic wounds. Wound Heal South Afr 1(2): 5-7.

3. Chakraborty S, Dutta TK, De A, Das M \& Ghosh S (2018): Impact of Bacterial Biofilm in Veterinary Medicine: An Overview. Int J Curr Microbiol App Sci 7(4): 3228-3239.

4. Rhoads DD, Wolcott R D \& Percival SL (2008). Biofilms in wounds management strategies. J Wound Care 17(11): 502508.

5. Manikandan C \& Amsath A (2013). Antibiotic susceptibility of bacterial strains isolated from wound infection patients in Pattukkottai Tamilnadu India. Int J Curr Microbiol App Sci 2(6): 195203.

6. Gautam R, Acharya A, Nepal HP \& Shrestha S (2013). Antibiotic susceptibility pattern of bacterial isolates from wound infection in Chitwan Medical College Teaching Hospital Chitwan. Nepal Int J Biomed Adv Res 4(2): 248-252.

7. Tayfour MA, Al-Ghamdi SM \& AlGhamdi AS (2005). Surgical wound infections in King Fahad Hospital at AlBaha. Saudi Med J 26(8): 1305-1306.

8. Rubin RH (2006). Surgical wound infection epidemiology pathogenesis diagnosis and management. BMC Infect Dis 6(1): 171.

9. Wegener HC, Aarestrup FM, Jensen LB, Hammerum AM \& Bager F (1999). Use of antimicrobial growth promoters in food animals and Enterococcus faecium resistance to therapeutic antimicrobial drugs in Europe. Emerg Infect Dis 5(3): 329.

10. Prescott JF, Hanna WB, Reid-Smith R \& Drost K (2002). Antimicrobial drug use and resistance in dogs. Canad Vet $J$ 43(2): 107.

11. Pak SI, Han HR \& Shimizu A (1999). Characterization of methicillin resistant Staphylococcus aureus isolated from dogs in Korea. J Vet Med Sci 61(16): 1013-1018.

12. Sani RA, Garba SA, Oyewole OA \& Ibrahim A (2012). Antibiotic resistance profile of gram positive bacteria isolated from wound infections in Minna Bida, Kontagora and Suleja area of Niger State. J Health Sci 2(3): 19-22.

13. Anguzu JR \& Olila D (2007). Drug sensitivity patterns of bacterial isolates from septic post-operative wounds in a regional referral hospital in Uganda. Afr Health Sci 7(3): 148-154.

14. Nelson LL (2011). Surgical site infections in small animal surgery. Vet Clin North Am Small Anim Pract 41(5): 1041-1056.

15. Saverino KM \& Reiter AMD (2018). Clinical presentation, causes, treatment, and outcome of lip avulsion injuries in dogs and cats: 24 cases (20012017). Front Vet Sci 5:144.

16. Bauer A, Kirby M, Sheris J \& Turek M (1966). Antibiotic susceptibility testing by a standardized single disk method. Am J Clin Pathol 45(4): 493-496.

17. Kalhoro DH, Luo S, Xie X, Zhao YB, Lu CP \& Liu YJ (2015). Streptococcus pluranimalium isolated from a canine respiratory case: identification and experimental infection in mice. Pak Vet J 35: 388-390.

18. Adaszek L, Gorna M, Zietek J, Kutrzuba J \& Winiarczyk S (2009). Bacterial nosocomial infections in dogs and cats. Zycie Weterynaryjne 84(2): 805-808.

19. Attili AR, Cerquetella M, Pampurini F, Laus F, Spaterna A \& Cuteri V (2012). Association between enrofloxacin and $\mathrm{N}$-acetylcysteine in recurrent bronchopneumopathies in dogs caused 
by biofilm producer bacteria. J Anim Vet Adv 11(4): 462-469.

20. Griffin GM \& Holt DE (2001). Dog-bite wounds bacteriology and treatment outcome in 37 cases. J Am Anim Hosp Assoc 37(5): 453-460.

21. Kelly PJ, Mason PR, Els J \& Matthewman LA (1992). Pathogens in dog bite wounds in dogs in Harare Zimbabwe. Vet. Rec 131(20): 464-466.

22. Talan DA, Citron DM, Abrahamian FM, Moran GJ \& Goldstein EJ (1999). Bacteriologic analysis of infected dog and cat bites. $N$ Engl J Med 340(2): 8592.

23. Rahman MM, Biswas DB, Islam MM \& Islam MA (2003). Cultural sensitivity of septic wound in animals. Pak J Biol Sci 6(2): 741-744.

24. Padhy A, Mishra R, Behera SS, Sahu AR $\&$ Sahoo S (2014). Microbial profile of canine persistent wound infections. Vet World 7(4): 244-247.

25. Thomas R, Duke SE, Karlsson EK, Evans A, Ellis P, Lindblad-Toh K \& Breen M (2008). A genome assemblyintegrated $\operatorname{dog} 1 \mathrm{Mb}$ BAC microarray a cytogenetic resource for canine cancer studies and comparative genomic analysis. Cyto Genone Res 122(2): 110121.

26. Urumova V, Chaprazov TS, Lyutskanov M \& Borisov I (2012). Microbiological analyses of canine infected wounds. Revue Méd Vét 163(4): 201-205.

27. Masaadeh HA \& Jaran AS (2009). Incident of Pseudomonas aeruginosa in post-operative wound infection. Am $J$ Infect Dis 5(1): 1-6.

28. Westgate SJ, Percival SL, Knottenbelt DC, Clegg PD \& Cochrane CA (2011). Microbiology of equine wounds and evidence of bacterial biofilms. Vet Microbiol 150(1-2): 152-159.

29. Schmidt, VM, Pinchbeck G, McIntyre KM, Nuttall T, McEwan N, Dawson S \& Williams NJ (2018). Routine antibiotic therapy in dogs increases the detection of antimicrobial-resistant faecal Escherichia coli.J Antimicrob Chemother doi:10.1093/jac/dky352.

30. Molchanova, N, Hansen P.R, Damborg P \& Franzyk, H (2018). Fluorinated antimicrobial lysine-based peptidomimetics with activity against methicillin-resistant $S$. pseudintermedius. J Pep Sci 24(7): e3098.

31. Kalhoro DH, Kalhoro MS, Pirzado SA, Kaka A, Tunio AN \& Parveen F (2016). Minimum inhibitory concentration of metronidazole against vaccinal strain of Pasteurella multocida. Sci Int (Lahore) 141(2): 37-40.

32. Kalhoro $\mathrm{DH}$, Kalhoro $\mathrm{AB}$, Rind $\mathrm{R}$, Kalhoro S \& Baloch H (2010). In vitro antimicrobial susceptibility of Pasteurella multocida.Pak J Agric Agric Eng Vet Sci 26(2): 80-86.

33. Bassessar V, Verma $Y$ \& Swamy M (2013). Antibiogram of bacterial species isolated from canine pyometra. Vet World 6(8): 546.

34. Maity S, Sarkar S \& Saha T (2009). Bacteriology and antibiogram of canine pyometra. Ind Vet J 86(9): 896-897.

35. Shambulingappa BE, Manegar GA \& Ananda KJ (2010). Study on Aerobic Bacterial flora in Canine abortions. Vet World 3(3): 111.

36. Roy SK, Das B \& Batabyal K (2009). Antibiogram of pathogenic Escherichia coli isolated from canine pyometra cases. J Interacad 13(4): 481-483. 